AUDIT

\title{
How valuable is ascitic cytology in the detection and management of malignancy?
}

\author{
R O S Karoo, T D R Lloyd, G Garcea, H D Redway, G S R Robertson
}

Postgrad Med J 2003;79:292-294

See end of article for authors' affiliations

Correspondence to: Mr T D R Lloyd, Department of Surgery, Secretary's Office, Old Ward 16, Leicester General Hospital Gwendolen Road, Leicester LE5 4PW, UK tdlloyd@ntlworld.com

Submitted

30 December 2002

Accepted

24 January 2003

\begin{abstract}
Introduction: Ascitic cytology is often requested in the early stages of ascitic assessment. A review of this practice in a major English teaching hospital is presented.

Method: Patients were retrospectively identified using the histopathology and patient administration system between January 1999 and May 2001.

Results: Of 276 samples sent for assessment 35 cases were found to be negative when on further review an intra-abdominal malignancy was present. The malignancy was diagnosed using a radiological modality. The sensitivity of ascitic cytology was found to be $60 \%$ with $100 \%$ specificity. A delay of up to five days could be incurred awaiting the cytology results before further radiological examinations were undertaken.

Conclusion: Too much hope is placed on ascitic cytology to provide the diagnosis at the expense of other investigations. It is recommended that the initial assessment should concentrate on history, examination, and basic tests on ascitic fluid to assess the serum-ascites albumin gradient. Ovarian malignancy is the only tumour type yielding a significant rate of detection from cytology with some prognostic impact. Results should not be awaited before abdominal ultrasound is undertaken. This more directed practice would help reduce unnecessary workload for the pathologist and has resource implications.
\end{abstract}

A cites refers to an increased volume of fluid collecting within the peritoneal cavity. It has been traditionally classified into a transudate or exudate depending on the protein content of the fluid. This has now been superseded by the serum-ascites albumin gradient (SAAG), which is calculated by subtracting the ascitic fluid albumin content from that of the serum albumin. ${ }^{1}$ A gradient greater than 11 $\mathrm{g} / \mathrm{l}$ can be caused by portal hypertension, heart failure, cirrhosis, hepatitis, massive metastatic disease, or Budd-Chiarri syndrome. A gradient less than $11 \mathrm{~g} / \mathrm{l}$ is commonly associated with carcinomatosis, pancreatic ascites, tuberculosis, and nephrotic syndrome.

It was noted in our experience that although a number of patients with significant ascites were found to have an intraabdominal malignancy, not one of these patients had their malignancy diagnosed by ascitic cytology. This anecdotal finding prompted a literature search and retrospective audit of ascitic cytology to determine its usefulness in managing patients with ascites.

\section{PATIENTS AND METHODS}

From January 1999 to May 2001, all ascitic fluid samples sent for cytology were identified using the Histopathology APEX computer system. Each report was reviewed and the number of pathology specimens (not just ascites) for each individual patient noted. Each patient's electronic record was also reviewed via the patient administration system and their coding diagnosis, investigations, operations, along with the consultant in charge of their care recorded. Those who were found to have an intra-abdominal malignancy by using these two methods but with a negative result on paracentesis cytology were further investigated by case note review and by the computerised radiology reporting system.

\section{RESULTS}

Over the 28 month period reviewed, 276 specimens of ascitic fluid from 239 different patients ( 87 male, 152 female) were analysed by a university teaching hospital in the UK. Of these 276 ascitic cytology specimens 48 (17\%) were found to contain malignant cells. Malignant cells that were ovarian in origin were found in $41(85 \%)$ patients of the positive samples analysed. The remaining positive specimens showed that three patients had lymphoma, one breast metastasis, one carcinoid, and two had an unknown primary. These 48 positive specimens were collected from 48 different patients, three of whom were male and 45 female.

Of the remaining 228 specimens from 192 patients (of which 84 were male, 108 female), 220 were reported has having no evidence of malignancy and another eight to be inadequate samples. However it was found that the results of 35 patients (19\%) from this group were false negatives and they did indeed have intra-abdominal cancer detected by some other modality.

Three sets of notes were unobtainable and therefore excluded from further statistical analysis, leaving 32 patients, nine male, 23 female. Thirteen of these patients had colorectal cancer, 10 gynaecological malignancies, eight liver metastases, and one lymphoma (fig 1). Of these 32 patients, nine samples of ascitic fluid were sent at the time of surgery, the operative notes clearly stating that signs of carcinoma were present. In all these cases the diagnosis had been made before surgery using radiological imagining. The sensitivity and specificity of ascitic fluid cytology is illustrated in table 1 .

In five of the false negative cases the initial investigation after presentation was an ascitic fluid aspiration. In three of these cases an ultrasound had been performed just before or at the same time as aspiration; however, it had been deemed to be non-diagnostic and there was a delay of four days on average incurred before computed tomography. In the other two patients delays of three and four days were incurred while awaiting the cytology result before an ultrasound was performed, at the request of the radiologist.

Table 2 shows the specialty requesting the investigation. A total of 75 out of 77 samples (97.5\%) sent from the physicians for cytology showed that no malignant cells were present (the 
Table 1 Sensitivity and specificity of ascitic fluid cytology

\begin{tabular}{|c|c|c|}
\hline Ascitic fluid & Sensitivity (\%) & Specificity (\%) \\
\hline Males ( $n=87$ ) & $3 / 12$ (25) & 100 \\
\hline Females $(n=192)$ & $45 / 68(66)$ & 100 \\
\hline Total number of samples $(n=276)$ & $48 / 80(60)$ & 100 \\
\hline
\end{tabular}

Three men and 45 women with positive cytology. Nine men and 23 women with false negative cytology

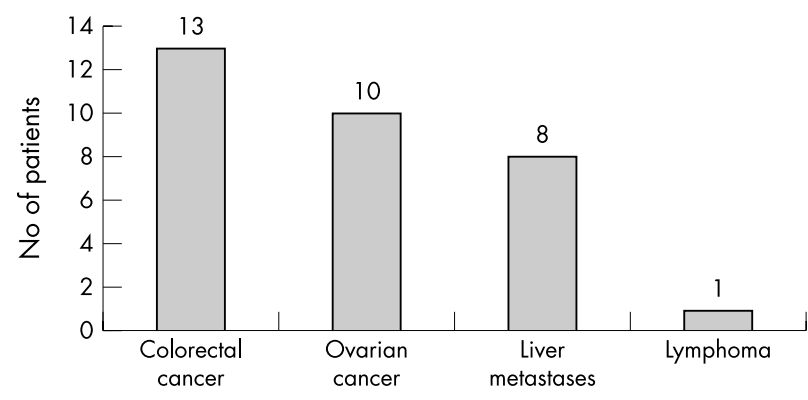

Figure 1 Diagnosis of the false negative aspirates.

Table 2 Specialty requests for cytology

\begin{tabular}{llll}
\hline Specialty & $\begin{array}{l}\text { No (\%) with } \\
\text { malignant cells }\end{array}$ & $\begin{array}{l}\text { No (\%) with no } \\
\text { malignant cells }\end{array}$ & $\begin{array}{l}\text { No (\%) with } \\
\text { insufficient } \\
\text { sample }\end{array}$ \\
\hline Medicine & $2(2.5)$ & $73(93)$ & $2(2.5)$ \\
Surgery & $9(21)$ & $32(76)$ & $1(3)$ \\
Gynaecology & $29(25)$ & $81(71)$ & $4(3.5)$ \\
Haematology & $4(20)$ & $15(75)$ & $1(5)$ \\
Oncology & $5(22)$ & $18(78)$ & 0 \\
\hline
\end{tabular}

remaining $2.5 \%$ showing ovarian malignancy). Specimens from gynaecology patients showed a $25 \%$ positive return rate and in each case the malignancy proved to be gynaecological in origin. The general surgeons found malignant cells in $21 \%$ of the samples sent, $89 \%$ of which were gynaecological in origin. All the false negatives came from surgical or gynaecological patients.

\section{DISCUSSION}

The cause of ascites is a common diagnostic challenge to many medical specialties. In the developed world approximately $80 \%$ of these patients will have underlying cirrhosis, while $10 \%$ are usually due to malignant causes. ${ }^{1}$ In assessing such patients sending ascitic fluid for cytology, microscopy, and albumin levels has been undertaken routinely. This approach, normally performed by a junior member of the team could, in many cases, be deemed unnecessary, and lack directed reasoning.

Our review found that $17 \%$ of ascitic fluid aspirates proved positive for malignant cells; with a sensitivity rate of $60 \%$ this compares well to that of other published studies. ${ }^{2-4}$ The cytological specimens reviewed yielded a false negative rate of $19 \%$. Runyon et al reported peritoneal carcinomatosis to be the only reason for a positive cytology, which occurs in approximately two thirds of patients with cancer and ascites. ${ }^{5}$ False negatives are therefore a significant problem if this single test is to be relied upon. With this uncertainty, when should ascitic fluid be sent for cytology and how should the patient be assessed?

The literature states that the initial management of the patient presenting with ascites should be a thorough history with examination, and this should then direct any further laboratory assessment, with cell count and differential, albumin, and culture being mandatory. In addition, serum albumin should be obtained to calculate the SAAG as an important determinate of the differential count. Cytology is to be performed only when there is a suspicion of malignancy. ${ }^{16}$ Runyon suggested the following three criteria as indications to proceed with cytology: (i) a history of cancer, (ii) no physical findings suggestive of liver disease, and (iii) an initial ascitic fluid sample with a high lymphocyte count $\left(500\right.$ cells $/ \mathrm{mm}^{3}$ ) and few neutrophils. ${ }^{7}$

In our study, excluding the false negatives and inadequate samples, 184 specimens (67\% of the total) were sent for assessment on patients who did not have malignancy. It may be assumed that this is due to the wide range of specialties within the hospital, and the lack of a protocol in place. A good proportion of these specimens would probably not have been sent for cytological analysis if the above criteria had been followed. Each cytological assessment costs approximately £30 (pounds sterling) and places an extra strain on not only the pathologists but also the technicians' time.

If there is a doubt regarding a definitive diagnosis, particularly in a female, a case can be made for sending cytology early. Thirty percent of woman with ovarian malignancy will have ascites at the time of diagnosis, ${ }^{8}$ and have a median survival of 10 months if untreated, but with appropriate chemotherapy a $50 \%$ five year disease-free survival can be achieved. .' Gastrointestinal tumours with ascites have a significantly poorer prognosis ${ }^{10}$ with a median survival of 10 weeks for pancreatic, 12 weeks for gastric, and 16 weeks for colorectal malignancy. ${ }^{11}$ It is, therefore, important to identify these patients early to start appropriate treatment. Some authors have commented that if ascites is thought to be secondary to tumour in a male or from a non-ovarian primary in females, that no further invasive investigation is warranted as it will not make any difference to the patient's long term survival. ${ }^{12} 13$

In our study ascitic fluid analysis for woman proved useful in the assessment of possible ovarian cancer. We found $85 \%$ $(\mathrm{n}=4 \mathrm{l})$ of all the positive cytology results were ovarian in origin. Other studies vary in this percentage from $7 \%-36 \% .{ }^{52-15}$ Our results are considerably higher and probably reflect the case mix at the different centres studied. Monte concluded that ovarian cancer is the most likely diagnosis in woman with a non-liver related ascites ${ }^{14}$ and Shen-Gunther and Manuel found that the presence of a pelvic mass and ascites was highly predictive of ovarian malignancy. ${ }^{16}$ In contrast only 12 men had malignant ascites of which nine were false negatives in comparison with 45 women with 23 false negatives.

The study has also highlighted the role of intraoperative ascitic fluid collection for analysis. In the management of ovarian cancer the detection of malignant cells in ascitic fluid is important for staging and has been found to have prognostic significance. ${ }^{17}$ For other intra-abdominal malignancies ascites is associated with a poorer prognosis, but it is not incorporated in the TNM classification. It has been published that for gastric, colorectal, and pancreatic carcinoma that the findings of positive cytology after peritoneal lavage may identify patients more likely to get recurrences. ${ }^{918}{ }^{19}$ However, there are no local or national guidelines for this to be used as a routine part of assessment for patients undergoing surgery for gastrointestinal malignancy.

The role of ultrasound has been found to have an important role in the investigation of potentially malignant ascites. In 19 of the 32 patients with false negative results the provisional diagnosis was made on ultrasound. We found that the hope placed in cytology providing the diagnosis caused a delay of up to five days before radiological imaging was performed.

From the results of our study we would recommend the following as a protocol on patients requiring investigation of ascites: 


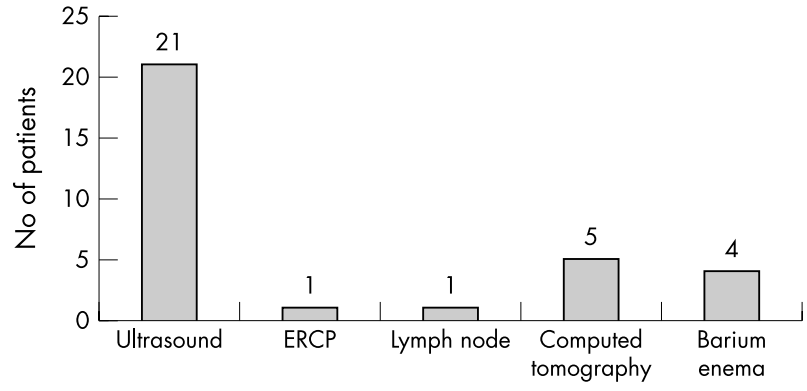

Figure 2 Diagnostic methods for false negative ascitic cytology

- Firstly, send cytology samples only on women, as this is the only group that showed a significant detection rate and a positive result that could alter management.

- Secondly, measure ascitic and serum albumin in the initial paracentesis to aid diagnosis if the history and examination are not conclusive, or suspicious of malignancy, rather than sending "routine" cytology.

- Thirdly, proceed to early ultrasound in suspected malignant ascites rather than awaiting cytology (fig 2).

As well as the economic benefits, this protocol could potentially have a significant clinical impact on the speed and accuracy of diagnosing the underlying cause of ascites.

\section{Authors' affiliations}

R O S Karoo, Department of Biomedical Sciences, Plastic Surgery and Burns Unit, University of Bradford, Bradford, West Yorkshire

T D R Lloyd, G Garcea, H D Redway, Leicester General Hospital Leicester

G S R Robertson, Leicester Royal Infirmary, Leicester

\section{REFERENCES}

1 McHutchinson JG. Differential diagnosis of ascites. Sem Liver Dis 1997; 17:191-201.

2 Motherby $\mathrm{H}$, Nadjari B, Friegel $\mathrm{P}$, et al. Diagnostic accuracy of effusion cytology. Diagn Cytopathol 1999;20:350-7.

3 Castaldo G, Oriani G, Cimino L, et al. Total discrimination of peritoneal malignant ascites from cirrhosis- and hepatocarcinoma-associated ascites by assays of ascitic cholesterol and lactate dehydrogenase. Clin Chem 1994:40: 478-83.

4 Colli A, Buccino G, Cocciolo $M$, et al. Diagnostic accuracy of sialic acid in the diagnosis of malignant ascites. Cancer 1989;63:912-16.

5 Runyon BA, Hoefs JC, Morgan TR. Ascitic fluid analysis in malignancy-related ascites. Hepatology 1988;5:1104-9.

6 Habeeb KS, Herrera JL Management of ascites: paracentesis as a guide. Postgrad Med 1997;101:191-8.

7 Runyon BA. Malignancy-related ascites and ascitic fluid "Humoral tests of malignancy" [editorial]. J Clin Gastroenterol 1994;18:94-8.

8 Enck RE. Malignant ascites. Am J Hosp Palliat Care 2002;19:7-8.

9 Hase K, Ueno H, Kuranaga N, et al. Intraperitoneal exfoliated cancer cells in patients with colorectal cancer. Dis Colon Rectum 1997:8: 1 134-40.

10 Blair SL, Chu DZJ, Schwartz RE. Outcome of palliative operations for malignant bowel obstruction in patients with peritoneal carcinomatosis from nongynecological cancer. Ann Surg Oncol 2001;8:632-7.

11 Parsons SL, Watson SA, Steele RJC. Malignant ascites. Br J Surg 1996:83:6-14.

12 Parsons SL, Lang MW, Steele RJC. Malignant ascites: a 2-year review from a teaching hospital. Eur J Surg Oncol 1996;22:237-9.

13 Garrison RN, Kaelin LD, Heuser LS, et al. Malignant ascites: clinical and experimental observations. Ann Surg 1986;203:644-51.

14 Monte SA, Ehya H, Lang WR. Positive effusion cytology as the initial presentation of malignancy. Acta Cytol 1987;31:448-52.

15 Ringberg QS, Doll DC, Loy TS, et al. Malignant ascites of unknown origin. Cancer 1989;64:753-5.

16 Shen-Gunther J, Mannel RS. Ascites as a predictor of ovarian malignancy. Gynecol Oncol 2002;87:77-83.

17 Simojoki M, Santala M, Vuopala S, et al. The prognostic value of peritoneal cytology in ovarian cancer. Eur J Gynaecol Oncol 1999;20:357-60

18 Ribeiro U, Gama-Rodriques JJ, Safatle-Ribeiro AV, et al. Prognostic significance of intraperitoneal free cancer cells obtained by laparoscopic peritoneal lavage in patients with gastric cancer. J Gastrointest Surg 1998:2:244-9

19 Konishi M, Kinoshita T, Nakagohri T, et al. Prognostic value of cyłologic examination of peritoneal washings in pancreatic cancer. Arch Surg 2002; 137:475-80. 